\title{
Psychological Constructs and Dysfunctional Eating in Pakistani University Students
}

\author{
Maryam Hussain*
}

\begin{abstract}
The present study aims to test a model that seeks to explore if parental bonding (mother care, father care, mother overprotection, and father overprotection) and loneliness are associated with the development of dysfunctional eating patterns such as cognitive restraint, emotional eating, uncontrolled eating, drive toward thinness, bulimia, and dissatisfaction with body image. It was further hypothesized that metacognitions, core beliefs and metaemotions could mediate between loneliness, parental bonding, and dysfunctional eating patterns. Correlational research design was used. A sample of 210 university students was selected from government and private universities in Pakistan. Urdu-translated versions of the UCLA Loneliness Scale (Version 3), Three Factor Eating Questionnaire-R-18, Eating Disorders Inventory-3, Parental Bonding Inventory, Beliefs about Emotions Scale, Eating Disorders Belief Questionnaire, and Metacognitions Questionnaire were used for assessment purposes. Correlational Analysis and Multiple Hierarchical Regression were used to analyze the data. Almost all dimensions of Parental Bonding showed significant relationship with some patterns of Dysfunctional Eating. Loneliness did not show any significant correlation with Dysfunctional Eating. No mediation hypotheses were supported yet some interesting relationships emerged. The findings of this research may help in understanding cultural contexts in a Pakistani sample. Some possible reasons why results could not support the previous literature are discussed.
\end{abstract}

$$
\begin{gathered}
\text { Keywords } \\
\text { undergraduates } \bullet \text { university students } \bullet \text { dysfunctional eating } \bullet \text { core beliefs } \\
\cdot \text { meta-emotions } \bullet \text { metacognitions } \bullet \text { loneliness } \bullet \text { parental bonding }
\end{gathered}
$$

\section{Introduction}

The role of psychological constructs has long been established in the initiation and sustainment of eating disorders. On the continuum of healthy eating behavior, either of the extremes

\footnotetext{
*University of Management and Technology, Sialkot, Pakistan maryamhussainqureshi@yahoo.com

doi: $10.3998 / \mathrm{jmmh} .238$

Conflicts of interest:

The author has no conflicts of interest to disclose.
} 
can be detrimental to an individual's health; for instance, obese males between the ages 20 and 30 can expect a $22 \%$ reduction in their lifespan (Fontaine et. al., 2003), whereas at the other extreme $20 \%$ adults with anorexia nervosa commit suicide (Arcleus, Mitchell, Wales, \& Nielsen, 2011). Some evidence from Pakistan has shown incidence of disordered eating patterns. Chohan and Kausar (2015) hypothesized that there is likely to be an association between metacognitions, meta-emotions, and dysfunctional eating. It was also hypothesized that gender will likely moderate the relationship among the aforementioned variables and that there will be gender differences. The sample of their study comprised 200 university students from two public sector universities of Lahore, Pakistan. The findings of this study revealed that emotional eating had a significant positive association with cognitive confidence and cognitive consciousness in male participants and with cognitive confidence and invalid emotions in female participants. Regarding the moderation hypothesis, it was found that gender does not act as a moderator among the association between metacognitions, meta-emotions, and dysfunctional eating. The prevalence and incidence of disordered eating patterns in Pakistan are more frequent as compared to fully diagnosed eating disorders. The results of a study conducted by Muazzam and Khalid (2008) found that some sort of disordered eating behavior is manifested among 45 to 50 percent of Pakistani college students.

The present research aims at finding the relationship between several different psychological constructs, such as parental bonding and loneliness and patterns of dysfunctional eating such as restrictive, disinhibited, or emotional eating. There are also measures that are characteristic of eating disorders; these include a person's drive to thinness, the proportion of a person to engage in bulimic behavior (i.e., bingeing and purging) and a person's dissatisfaction with his or her body image. The psychological variables that may be associated with dysfunctional eating behavior include metacognitions (thoughts about thoughts), meta-emotions (thoughts about emotions; Sorenson \& Strodl, 2015), specific core beliefs (Cooper, Rose, \& Turner, 2006), and parental bonding (Turner, Rose, \& Cooper, 2005a). Furthermore, it has also been observed that the association between parental bonding and dysfunctional eating is mediated by core beliefs (Turner, Rose, \& Cooper, 2005b). The variable of loneliness has been found to be linked with eating disorders (American Psychiatric Association, 2000). The present study also aims to look for the relationship between loneliness and dysfunctional as well as disordered eating in a Pakistani sample.

\section{Parental Bonding and Dysfunctional Eating}

Research literature suggests that unhealthy patterns of attachment and bonding between children and their parents is pivotal in developing and upkeep of eating disorders (Heesacker \& Neimeyer, 1990). For instance, Walters and Kendler (1995) in their study including more than 2000 female twins concluded that high maternal overprotectiveness was significantly related to anorexia. An association was shown with bulimia and high parental rejection (Stuart, Laraia, Ballenger, \& Lydiard, 1990), less paternal affection, and more paternal control toward them as compared to their siblings (Wonderlich, Ukestad, \& Perzacki, 1994). However, while these studies postulate that perceived parental overprotection and care might be significant in the etiology of disordered eating, the possible psychosocial factors playing a role in this relationship are yet to be discovered. Findings discussed above suggest that family experiences may be associated with developing unhealthy core beliefs, which can further affect pathological eating (Leung et al., 2000).

\section{Loneliness and Dysfunctional Eating}

A number of studies have demonstrated that early experiences with loneliness in childhood may set the stage for developing eating disorders. A study found women having a history of 
anorexia (binge/purge type) and bulimia reported of experiencing more intense feelings of loneliness in their childhood as compared to controls (Troop \& Bifulco, 2002). According to the DSM- IV Text Reivsed (APA, 2000), there has been a dearth of studies assessing the relationship between disordered eating patterns and loneliness. Among these limited studies, the majority focus on the relationship between binge eating and loneliness. Studies have concluded that such a relationship mostly exists in individuals diagnosed with bulimia (Masheb \& Grilo, 2006). According to Tuschen and Vogele (1999), feelings of loneliness in restrained eaters often lead to higher food consumption and a desire to binge eat in patients with bulimia nervosa.

Disordered eating is defined by the DSM-IV as "a wide range of irregular eating behaviors that do not warrant a diagnosis of a specific eating disorder". The phrase disordered eating is defined as "those problematic eating behaviors, which may include bingeing, purging, or restrictive dieting, that are less in intensity as compared to those necessary to meet the criteria outlined for diagnosing a defined eating disorder according to internationals standards". Despite the detrimental role of dysfunctional eating in general health, the effectiveness of psychological interventions is still modest; the reason being that the psychological mechanisms underpinning dysfunctional eating are still ambiguous and unclear in nature.

Therefore, the rationale of the study is to assess a predictive and mediating relationship between the literature backed psychological constructs in order to explain incidence of dysfunctional eating which will further help in the development of specific, problem oriented psychological interventions accordingly in Pakistani culture.

In light of the literature discussed above, it was hypothesized that there will be a significant relationship between parental bonding, loneliness, metacognitions, meta-emotions, core beliefs, and patterns of dysfunctional eating. It is also proposed that core beliefs, metacognitions, and meta-emotions will likely mediate between parental bonding (mother care, mother overprotection, father care, and father overprotection) and dysfunctional eating behaviors (drive to thinness, bulimic behavior, dissatisfaction with one's body image, disinhibited/ uncontrolled eating, restraint eating, and emotional eating).

\section{Method}

For the present study, correlational research design was used to investigate the predictors of eating behaviors. Out of a total sample of 217 undergraduate students, seven participants did not follow instructions and thus the included sample for the present study was reduced to 210 participants.

A formal approval was sought from the university's Departmental Doctoral Program Committee (DDPC). The data was collected from several different departments of University of the Punjab (PU), University of Management and Technology (UMT), and Government College University (GCU), Lahore. The study was conducted in 2016. No participant was coerced to participate; their consent was taken beforehand and complete debriefing was provided at the end of data collection.

\section{Participants}

Undergraduate Pakistani students were recruited, excluding international students as well as students with visual and/or auditory impairment and physical disabilities. The exclusion was solely done for the reason of increasing homogeneity in the sample. 


\section{Measures}

A demographic form developed by the researchers was administered to the participants to get their personal information such as age, gender, educational level, marital status, and parental income. Participants'weight and height were taken to calculate their BMI. The Parental Bonding Inventory (PBI) is a self-report questionnaire developed by Parker, Tupling, and Brown (1979) and is designed to measure the fundamental parental dimensions of 'care' and 'control/over-protection' for both parents separately. For the present study, an Urdu-translated version of PBI was used (Anwar \& Fatima, 2012).

The UCLA Loneliness Scale-Version 3 was used in this study. Developed by Russell and colleagues (1996) and translated into Urdu by Hussain and Kausar (2016) was used to measure the construct of loneliness. Other scales used to measure possible mediators include the Beliefs about Emotions Scale (BAES) developed by Rimes and Chalder (2010) and translated in Urdu by Chohan and Kausar (2015); the Eating Disorder Core Belief Questionnaire (Fairchild \& Cooper, 2010), translated into Urdu by Hussain and Rukhsana (2016); and metacognitions questionnaire (MCQ) Urdu-translated version.

Urdu-translated versions of the Eating Disorder Inventory (Garder 1991) and the Three Factor Eating Questionnaire R-18 (Karlsson, Persson, Sjostrom, \& Sullivan, 2000) were used to collect information regarding the dependent variables.

\section{Data Analyses}

Data were analyzed using the Statistical Package for the Social Sciences (version 20.0) to examine whether parental bonding, loneliness, and dysfunctional eating have any relationship and if metacognitions, core beliefs and meta-emotions mediate the relationship between parental bonding and dysfunctional eating. First Bivariate Pearson Moment Correlational Analysis was done and further mediation analysis was conducted using Multiple Hierarchical Regression analysis.

\section{Results}

The average age of participants was approximately 21 years old. The sample comprised mostly (around 65\%) female participants. The majority were enrolled in 4-year BS programs and attended the government university. Psychology majors made up the majority of the participants, followed by the students in information technology or computer sciences, business and accounting, and other disciplines. Nearly 90 percent of the sample were socioeconomically middle class socioeconomic status, and almost all the study participants (98\%), reported being single and unemployed. The majority belonged were from two-parent households and urban backgrounds. In survey materials, 93 to 95 percent of did not report any physical or eating related dysfunctions or disorders. Lastly, participant Body Mass Index (BMI) was calculated by using their weights and heights. Almost three-quarters of participants were in the normal BMI range.

\section{Relationship between demographics, loneliness, parental bonding and dysfunctional eating}

Bivariate Pearson Product Moment Correlation analysis was used to assess the relationship between demographic characteristics of the participants and patterns of dysfunctional eating. 


\begin{tabular}{|c|c|c|c|c|c|c|c|c|c|c|c|c|c|c|c|c|c|c|c|}
\hline ల్ల & $\hat{0}$. & 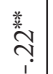 & $\exists$ & $\stackrel{\oplus}{\hookrightarrow}$ & ฮ̊. & 善 & 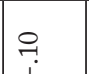 & gे & $\hat{o}$. & 8 & ø. & 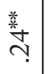 & $F$ & 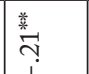 & $\stackrel{* 0}{\circ}$ & $F$ & $\hat{o}$ & to & $\stackrel{\infty}{0}$ \\
\hline$\widehat{\mathbf{N}}$ & $\because$ & in & 善 & $\stackrel{0}{0}$ & oे. & $\dddot{?}$ & $\vec{\sigma}$. & $\overrightarrow{0}$ & $\tilde{\rho}$ & 느. & t. & 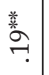 & $\sigma_{i}$ & $\stackrel{\oplus}{\rightarrow}$ & $\stackrel{\infty}{\circ}$ & oे & $\begin{array}{l}\infty \\
0\end{array}$ & $\tilde{\rho}$ & 祆 \\
\hline 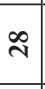 & oे. & $\begin{array}{l} \\
\text { i. } \\
i\end{array}$ & 丵 & بै & $\stackrel{*}{\stackrel{*}{\circ}}$ & 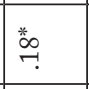 & $\vec{\sigma}$ & $\tilde{o}_{i}$ & $\stackrel{*}{+}$ & 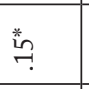 & 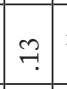 & 善 & 8 & 善) & 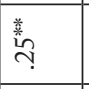 & 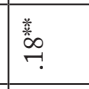 & \begin{tabular}{|l} 
\\
\end{tabular} & $\exists$ & 善 \\
\hline$\hat{\imath}$ & $\begin{array}{l}0 \\
i \\
i\end{array}$ & $\mid \begin{array}{l}0 \\
0 \\
i \\
1\end{array}$ & ธै. & $\hat{i}_{i}$ & $\underset{1}{7}$ & $F_{i}$ & ¿t. & $\underset{i}{8}$ & no & $\delta_{i}$ & $\begin{array}{l}t_{0} \\
+\end{array}$ & $\stackrel{2}{\circ}$ & ?ㅜ & L & \& & no & $\begin{array}{c}0 \\
i\end{array}$ & oे & 8 \\
\hline$\stackrel{\sim}{\mathrm{N}}$ & ๑ & 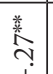 & 菐 & $\stackrel{.}{\stackrel{*}{\longrightarrow}}$ & $\stackrel{*}{\stackrel{*}{r}}$ & $\stackrel{*}{\stackrel{*}{\circ}}$ & $\stackrel{0}{0}$ & 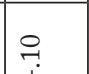 & ㄴ. & 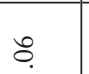 & t. & 善 & $\because$ & 善 & 善 & 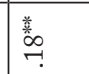 & $\stackrel{*}{*} \underset{\sim}{\circ}$ & ô. & 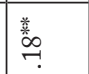 \\
\hline 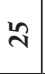 & $\because$ & $\because$ & o. & ơ. & \&. & 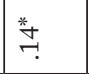 & $\hat{o}_{i}$ & 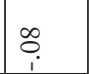 & ㄴo? & \&. & 8 & 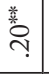 & $\stackrel{\infty}{0}$ & 丵 & $\stackrel{\infty}{\circ}$ & $\stackrel{0}{0}$ & $\tilde{c}$ & ô. & $\stackrel{\oplus}{\oplus}$ \\
\hline$\underset{\sim}{\Delta}$ & 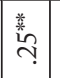 & 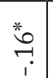 & 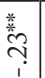 & 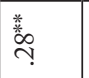 & 丵 & $\begin{array}{l}\text { 善 } \\
\text { के }\end{array}$ & $\tilde{\rho}$. & 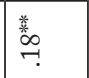 & $\begin{array}{l}\text { 業 } \\
\text { in }\end{array}$ & 善 & \begin{tabular}{|l|} 
橎 \\
\end{tabular} & 善 & $\overrightarrow{0}$ & $\hat{o}$ & 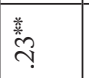 & 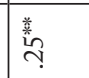 & 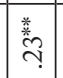 & 善 & 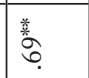 \\
\hline$\approx$ & $\mid \begin{array}{l}2 \\
0 \\
i\end{array}$ & ๆ & 畧 & $\underset{i}{\stackrel{2}{2}}$ & ò & $\hat{0}$ & $\hat{0}$ & 善 & 蟋 & 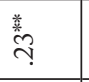 & 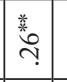 & $\begin{array}{c}\overrightarrow{0} \\
i \\
i\end{array}$ & $\stackrel{*}{\stackrel{2}{n}}$ & $\stackrel{*}{\stackrel{*}{\varphi}}$ & ô. & 8 & ò. & 善 & 帣 \\
\hline ส & $\because$ & $\begin{array}{l}n \\
n_{1} \\
i\end{array}$ & $\begin{array}{l}n \\
\text { ?. } \\
i\end{array}$ & $F$ & $\stackrel{*}{+}$ & 善 & $\stackrel{*}{*}$ & 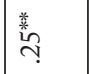 & 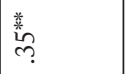 & 善 & 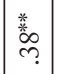 & $\mathbb{i}_{i}$ & 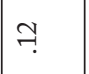 & 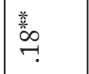 & $\tilde{o}$. & $\stackrel{\oplus}{\hookrightarrow}$ & $\underset{1}{7}$ & 善 & 善 \\
\hline$\vec{\nabla}$ & $\begin{array}{l}8 \\
\end{array}$ & \begin{tabular}{|l|} 
\\
0 \\
0 \\
1
\end{tabular} & 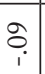 & $\stackrel{\varphi}{\hookrightarrow}$ & §ి. & Oे. & 恶 & 善 & $\tilde{o}_{i}$ & oे & 善 & | & 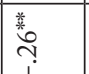 & 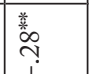 & 8 & 8 & $\underset{0}{\infty}$ & 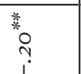 & ô. \\
\hline సి & 8 & \begin{tabular}{|l|} 
\\
\\
\\
\end{tabular} & 尊 & $\stackrel{0}{0}$ & 능. & $\stackrel{\infty}{0}$ & 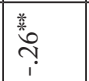 & 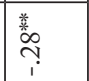 & $\tilde{D}_{i}$ & $\stackrel{* 0}{\dddot{1}}$ & 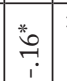 & 㽧 & 善 & $\begin{array}{l}.0 \\
7 \\
7\end{array}$ & $\stackrel{0}{0}$ & $\stackrel{\infty}{0}$ & 8 & 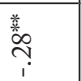 & $\begin{array}{l} \\
\frac{3}{7}\end{array}$ \\
\hline$\approx$ & 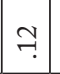 & $\begin{array}{l}0 \\
0 \\
i\end{array}$ & 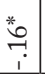 & 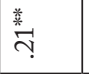 & $\stackrel{\text {.⿳䒑⿰幺幺 }}{\overbrace{}}$ & $\begin{array}{l}\text { 丵 } \\
\text { }\end{array}$ & 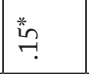 & \begin{tabular}{l}
$*$ \\
$\stackrel{*}{\infty}$ \\
\hdashline
\end{tabular} & 恶 & 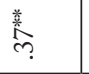 & 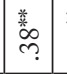 & 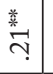 & o. & nn & $\stackrel{*}{\stackrel{*}{t}}$ & $F$ & $\stackrel{2}{\neg}$ & 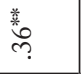 & ' \\
\hline$\stackrel{\infty}{\sim}$ & 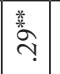 & 8 & $\stackrel{*}{\longrightarrow}$ & $\stackrel{*}{\hookrightarrow}$ & $\stackrel{0}{0}$ & $\begin{array}{l}\text { 善 } \\
\end{array}$ & بْ & 善 & 善 & $\begin{array}{l}\text { 善 } \\
\text { 号 }\end{array}$ & \begin{tabular}{|l|} 
善 \\
ले
\end{tabular} & $\stackrel{*}{+}$ & $\stackrel{*}{\sim}$ & $\hat{o}$ & 谷 & 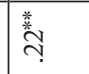 & 丵 & 1 & ' \\
\hline$\approx$ & $\hat{o}$ & $\stackrel{*}{*}$ & 葛 & $\begin{array}{l}\text { 善 } \\
\Rightarrow\end{array}$ & 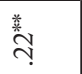 & ๆ & $\stackrel{\text { in }}{\mathrm{H}}$ & $F$ & $\stackrel{*}{\stackrel{*}{\hookrightarrow}}$ & $\stackrel{\oplus}{\hookrightarrow}$ & 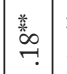 & $\begin{array}{l}\text { 善 } \\
\stackrel{0}{0}\end{array}$ & 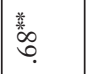 & 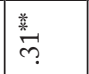 & 善 & 善 & 1 & , & ' \\
\hline$\stackrel{?}{-1}$ & 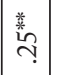 & \begin{tabular}{|l|} 
\\
$\vec{*}$ \\
$\vec{p}$
\end{tabular} & 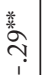 & 菐 & 善 & 鄯 & $\tilde{\sigma}$ & to. & 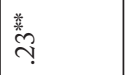 & $\stackrel{*}{\stackrel{*}{\varphi} !}$ & \begin{tabular}{|l|} 
\\
\\
7
\end{tabular} & 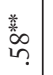 & 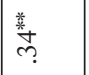 & 8 & $\begin{array}{l}\text { 善 } \\
\text { in }\end{array}$ & ' & 1 & ' & , \\
\hline$\stackrel{n}{2}$ & o. & 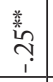 & 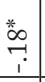 & $\stackrel{*}{\circ}$ & $\stackrel{\text { 丵 }}{\uparrow}$ & $\stackrel{*}{\stackrel{0}{\varphi}}$ & ¿ & 8 & ๆ & $\stackrel{\infty}{\circ}$ & $F$ & 憘 & 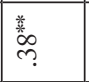 & $\tilde{c}$ & ' & ' & 1 & ' & \\
\hline \pm & $\left|\begin{array}{c}. \\
0 \\
0 \\
i\end{array}\right|$ & 啚 & 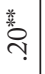 & $\stackrel{*}{*}$ & 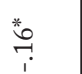 & $\stackrel{*}{*}$ & 恙 & $\stackrel{\text { 悬 }}{\sim}$ & $\overrightarrow{0}$ & $\vec{\sigma}$ & $\stackrel{\infty}{\circ}$ & $\underset{7}{\stackrel{*}{7}}$ & 羙 & ' & ' & ' & , & 1 & 1 \\
\hline 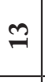 & $\stackrel{0}{0}$ & ㄴ. & t. & $\stackrel{\infty}{0}$ & $\stackrel{n}{o}$. & o. & 鄯 & 善) & 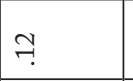 & $F$ & 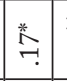 & 善 & ' & ' & ' & ' & , & 1 & \\
\hline$\approx$ & \begin{tabular}{ll}
\multirow{2}{*}{} \\
$\sim$
\end{tabular} & $\begin{array}{l}\text { 善 } \\
\text { ? }\end{array}$ & 羬 & 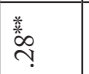 & $\begin{array}{l}\text { 善 } \\
\text { ले }\end{array}$ & 总 & 5 & $\tilde{o}$ & $\stackrel{\oplus}{\hookrightarrow}$ & 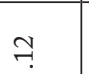 & $F$ & 1 & ' & 1 & ' & 1 & 1 & 1 & ' \\
\hline$=$ & 草 & 8 & 7 & $\hat{o}$. & $F$ & 善 & ب. & 善 & 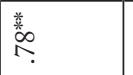 & $\begin{array}{l}\text { 恶 } \\
\infty \\
0 .\end{array}$ & , & & ' & ' & 1 & 1 & , & 1 & ' \\
\hline$\approx$ & \begin{tabular}{|c|c|} 
\\
\\
ì
\end{tabular} & 8 & $?$ & $\because$ & $\stackrel{\oplus}{\hookrightarrow}$ & 善 & $\begin{array}{l}\text { 教 } \\
\text { f. }\end{array}$ & $\begin{array}{l} \\
\end{array}$ & 善 & 1 & 1 & ' & ' & 1 & 1 & ' & , & 1 & \\
\hline$\sigma$ & 善 & $\begin{array}{l}\infty \\
0 \\
i\end{array}$ & ? & $\stackrel{*}{\stackrel{2}{?} ? ~}$ & 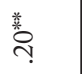 & $\begin{array}{l}\text { 善 } \\
\text { in } \\
\text { in? }\end{array}$ & $\stackrel{\text { 丵 }}{\Upsilon}$ & 善 & ' & 1 & , & , & , & 1 & 1 & 1 & , & 1 & ' \\
\hline$\infty$ & 8 & \begin{tabular}{|l|}
\multirow{H}{*}{} \\
\end{tabular} & $\hat{\mathrm{o}}$ & $\stackrel{*}{*}$ & $\underset{7}{7}$ & 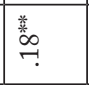 & 善 & 1 & ' & 1 & 1 & , & ' & 1 & ' & , & 1 & 1 & \\
\hline$\wedge$ & $\stackrel{0}{0}$ & 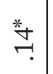 & 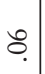 & $\hat{0}$ & $\tilde{c}$ & 晜 & ' & , & 1 & 1 & ' & ' & ' & ' & & ' & 1 & 1 & ' \\
\hline 0 & \begin{tabular}{|l|l|} 
\\
\end{tabular} & $\begin{array}{ll}* \\
\stackrel{*}{2} \\
7 \\
\end{array}$ & 尊 & 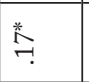 & 善 & ' & 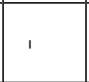 & , & ' & , & ' & , & ' & ' & & , & , & , & \\
\hline in & \begin{tabular}{|l|} 
善 \\
\end{tabular} & 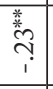 & $\begin{array}{l}\text { 普 } \\
7 \\
i \\
\end{array}$ & 善 & , & ' & ' & , & ' & , & ' & , & ' & 1 & I & 1 & , & 1 & ' \\
\hline$\nabla$ & 善 & $\begin{array}{c}\text { 善 } \\
\text { o. } \\
\end{array}$ & 曾 & ' & . & 1 & ' & , & ' & ' & 1 & I & ' & 1 & ' & , & , & 1 & \\
\hline$m$ & 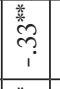 & $\begin{array}{l}\text { 丵 } \\
\text { in? }\end{array}$ & ' & ' & & 1 & ' & , & 1 & , & 1 & , & ' & 1 & ' & ' & 1 & 1 & ' \\
\hline$\sim$ & 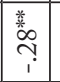 & & ' & ' & ' & 1 & ' & ' & 1 & 1 & 1 & , & ' & 1 & , & 1 & , & 1 & ' \\
\hline- & \begin{tabular}{|l|}
1 \\
\end{tabular} & 1 & I & 1 & 1 & 1 & 1 & 1 & 1 & 1 & 1 & I & 1 & 1 & I & 1 & 1 & 1 & 1 \\
\hline 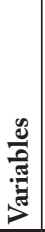 & 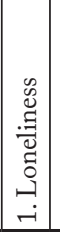 & 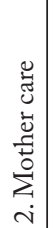 & 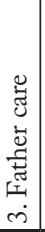 & 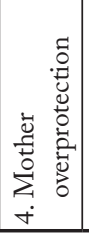 & 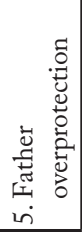 & 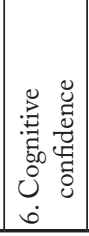 & 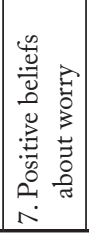 & 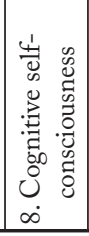 & 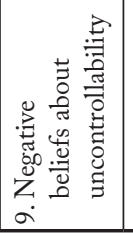 & 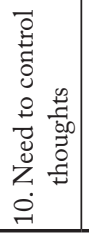 & 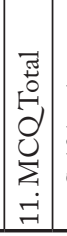 & 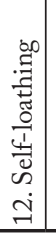 & 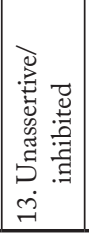 & 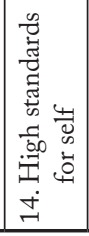 & 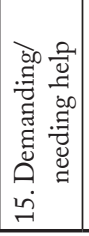 & 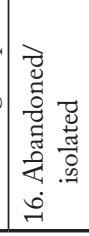 & 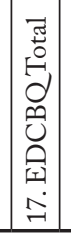 & 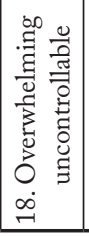 & 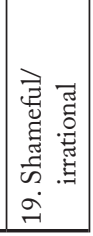 \\
\hline
\end{tabular}




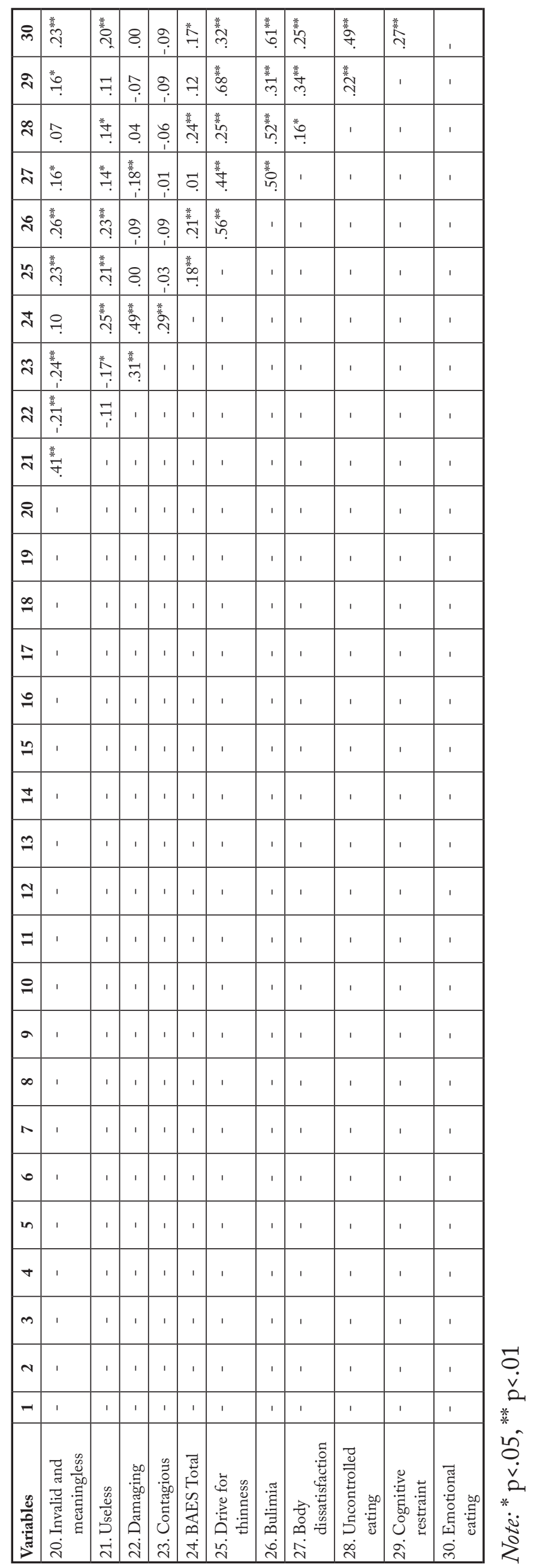


The BMI of the participants show a significant positive correlation with all patterns of dysfunctional eating except for uncontrolled eating. The bulimic pattern of dysfunctional eating also had significant positive correlation with BMI, and with the participants' university affiliation, whereas it has a significant negative correlation with gender. All other diversions of dysfunctional eating, except bulimia, show no significant relationships with any demographic characteristics of the participants except with their BMI.

Loneliness does not show any significant relationship with any of the dependent variables; rather, a new finding emerged wherein loneliness had a significant positive relationship with three dimensions (metacognition, core beliefs, and meta-emotions) which have been used as mediators in the present study. Only uncontrolled eating shows significant relationships with all four dimensions of parental bonding. Likewise, only bulimia shows significant relationships with all four dimensions of parental bonding.

\section{Metacognitions, core beliefs, and meta-emotions as mediators between loneliness and parental bonding}

A total of six mediational analyses have been performed to assess the hypotheses whether metacognitions, core beliefs, and meta-emotions mediated between loneliness and the four dimensions of parental bonding (mother care, mother overprotection, father care, and father overprotection) and several different patterns of dysfunctional eating (drive to thinness, bulimic behavior, dissatisfaction with one's body image, disinhibited/ uncontrolled eating, restraint eating, and emotional eating). The hierarchical regressions do not show significant mediation effects to be taking place in any of these analyses. Mediation tests have been carried out using the mother care, father care, mother overprotection, and father overprotection together as predictors, with total scores of metacognitions questionnaire, core-beliefs questionnaire and meta-emotions scale as mediators, and disinhibited/ uncontrolled eating, restraint eating, emotional eating, drive towards thinness, bulimic behavior, and dissatisfaction with one's body image separately as outcome variables.

The assumption of 'no or little multicollinearity' in multiple regression was not met for the present study; the predictors have been retained because the aim of the present study was to test a model of dysfunctional eating as a whole, and multicollinearity does not affect the overall fit of the model or produce bad predictions (Frost, 2013).

The following tables have been made separately for each step of mediation conducted through hierarchical multiple regression:

It can be observed in the tables that the paths that establish a significant relationship between the predictor and the dependent variables in the first step do not fulfill the conditions for mediation in the next steps. Most importantly, it can be observed in the second step wherein the predictor must be significantly associated with the hypothesized mediator that, except for 'father overprotection', no predictor shows any significant relationship with any of the mediators. But again, father overprotection does not show any significant relationship with any of the dependent variables in the first step, thus, making it impossible for all the mediation conditions to be fulfilled. Thus, it can be concluded from the results that metacognitions, meta-emotions, or core beliefs are not found to mediate between the four diversions of parental bonding and different patterns of dysfunctional eating.

\section{Discussion}

The present study aimed to find an association between parental bonding, loneliness, and dysfunctional eating patterns in a sample of undergraduate university students in Pakistan. Findings 


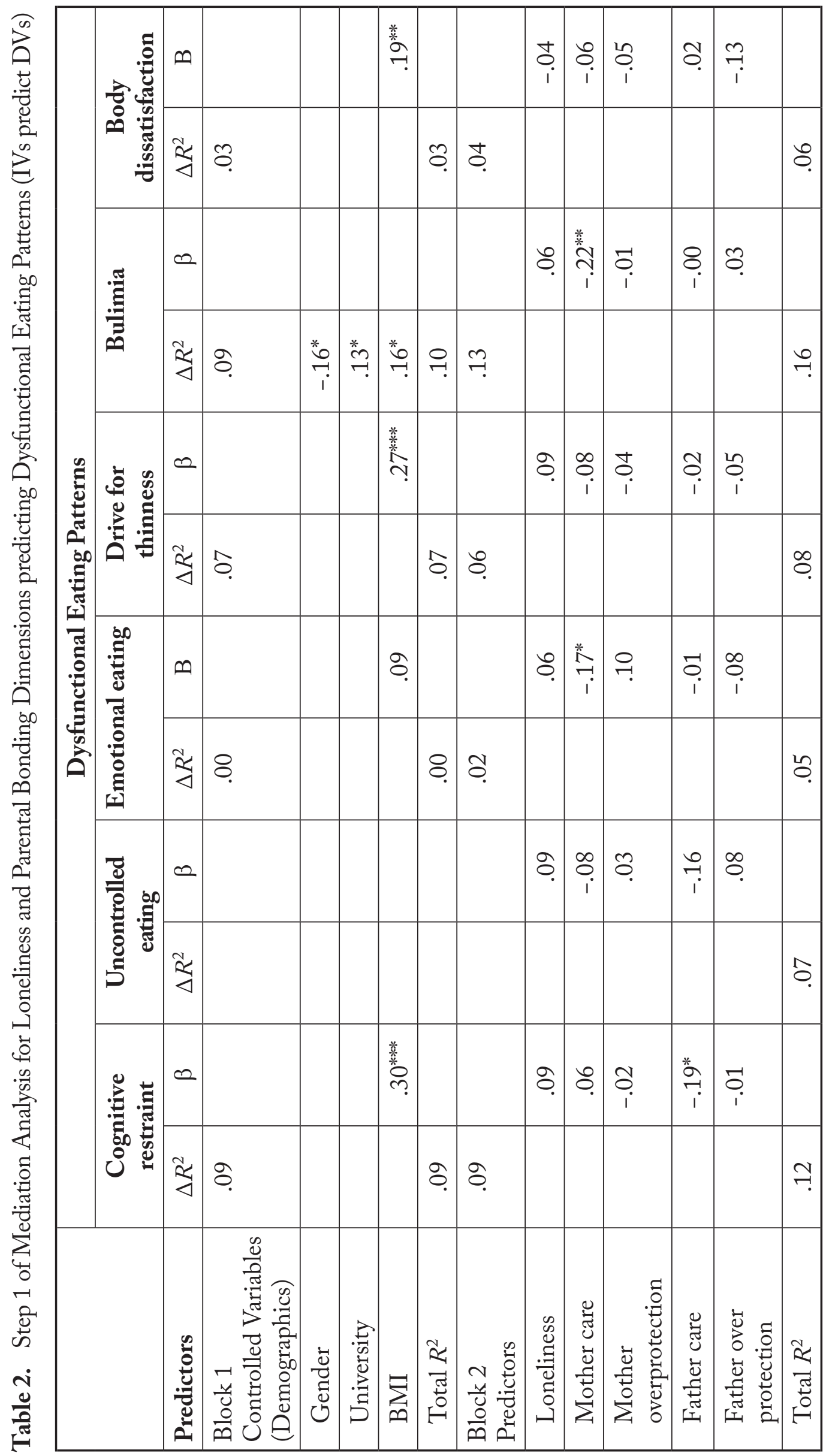


Table 3. Step 2 of Mediation Analysis for Loneliness and Parental Bonding Dimensions predicting Metacognitions, Meta-emotions, and Core Beliefs (IVs predict Mediators)

\begin{tabular}{|l|c|c|c|}
\hline & Meta cognitions & Meta-emotions & Core-beliefs \\
\hline Predictors: & $\beta$ & $\beta$ & $\beta$ \\
\hline Loneliness & $.15^{*}$ & .07 & $.25^{* * *}$ \\
\hline Mother care & .11 & .01 & -.09 \\
\hline Mother overprotection & .01 & $.22^{* *}$ & .04 \\
\hline Father care & -.14 & -.16 & -.02 \\
\hline Father over protection & .07 & .00 & .16 \\
\hline Total $R^{2}$ & .02 & .10 & .06 \\
\hline
\end{tabular}

Table 4. Step 3 of Mediation Analysis for Metacognitions, Meta-emotions, and Core Beliefs predicting Dysfunctional Eating Patterns (Mediators predict DVs)

\begin{tabular}{|l|c|c|c|c|c|c|c|c|c|c|c|c|}
\hline & \multicolumn{2}{|c|}{$\begin{array}{c}\text { Cognitive } \\
\text { restraint }\end{array}$} & \multicolumn{2}{|c|}{$\begin{array}{c}\text { Uncontrolled } \\
\text { eating }\end{array}$} & \multicolumn{2}{c|}{$\begin{array}{c}\text { Emotional } \\
\text { eating }\end{array}$} & \multicolumn{2}{c|}{$\begin{array}{c}\text { Drive for } \\
\text { thinness }\end{array}$} & \multicolumn{2}{|c|}{ Bulimia } & \multicolumn{3}{|c|}{$\begin{array}{c}\text { Body } \\
\text { Dissatisfaction }\end{array}$} \\
\hline Mediators: & $\beta$ & $\mathrm{R}^{2}$ & $\beta$ & $\mathrm{R}^{2}$ & $\beta$ & $\mathrm{R}^{2}$ & $\beta$ & $\mathrm{R}^{2}$ & $\beta$ & $\mathrm{R}^{2}$ & $\beta$ & $\mathrm{R}^{2}$ \\
\hline $\begin{array}{l}\text { Meta- } \\
\text { cognitions }\end{array}$ & .04 & .00 & .13 & .01 & .03 & .00 & .00 & .00 & .04 & .00 & -.04 & .00 \\
\hline $\begin{array}{l}\text { Meta- } \\
\text { emotions }\end{array}$ & .12 & .01 & $.24^{* *}$ & .05 & $.17^{*}$ & .02 & $.18^{* *}$ & .03 & $.21^{* *}$ & .04 & .01 & .00 \\
\hline $\begin{array}{l}\text { Core- } \\
\text { beliefs }\end{array}$ & .08 & .00 & $.19^{* *}$ & .03 & .07 & .00 & .03 & .00 & $.16^{*}$ & .02 & -.03 & .00 \\
\hline
\end{tabular}

of the present study revealed that mother care and father care dimensions of parental bonding have a significant negative correlation whereas mother overprotection and father overprotection dimensions of parental bonding have a significant positive correlation with bulimia and uncontrolled eating patterns of dysfunctional eating. The results suggest that, for this sample, parental care is low and parental overprotection is high in individuals with bulimia as well as those with uncontrolled eating tendencies. This result is supported by the existing literature since parenting practices have been found to influence miscellaneous aspects of children's behavioral and cognitive development, including disordered eating (Tata, Fox, \& Cooper, 2001). These results are also in line with a previous study evaluating the mediating role of character and temperament between parental bonding and bulimic symptoms in women (Fassino, Amianto, Rocca, \& Dagga, 2010). The results of 2010 study showed that bulimic individuals perceived low paternal and maternal care and self-directedness, disordered eating, and high novelty seeking. Maternal care was found to negatively correlate with social inadequacy, insecurity, and impulsiveness. Paternal care was found to predict self-directedness, novelty seeking, interoceptive awareness, asceticism, and impulsiveness. The mediating effect of self-directedness between paternal care and disordered eating was found to be significant, but not that of novelty seeking. Summed up, the 2010 study concluded that parental care is lower among their sample of bulimic women than in control groups even when controlled for possible confounding variables. 


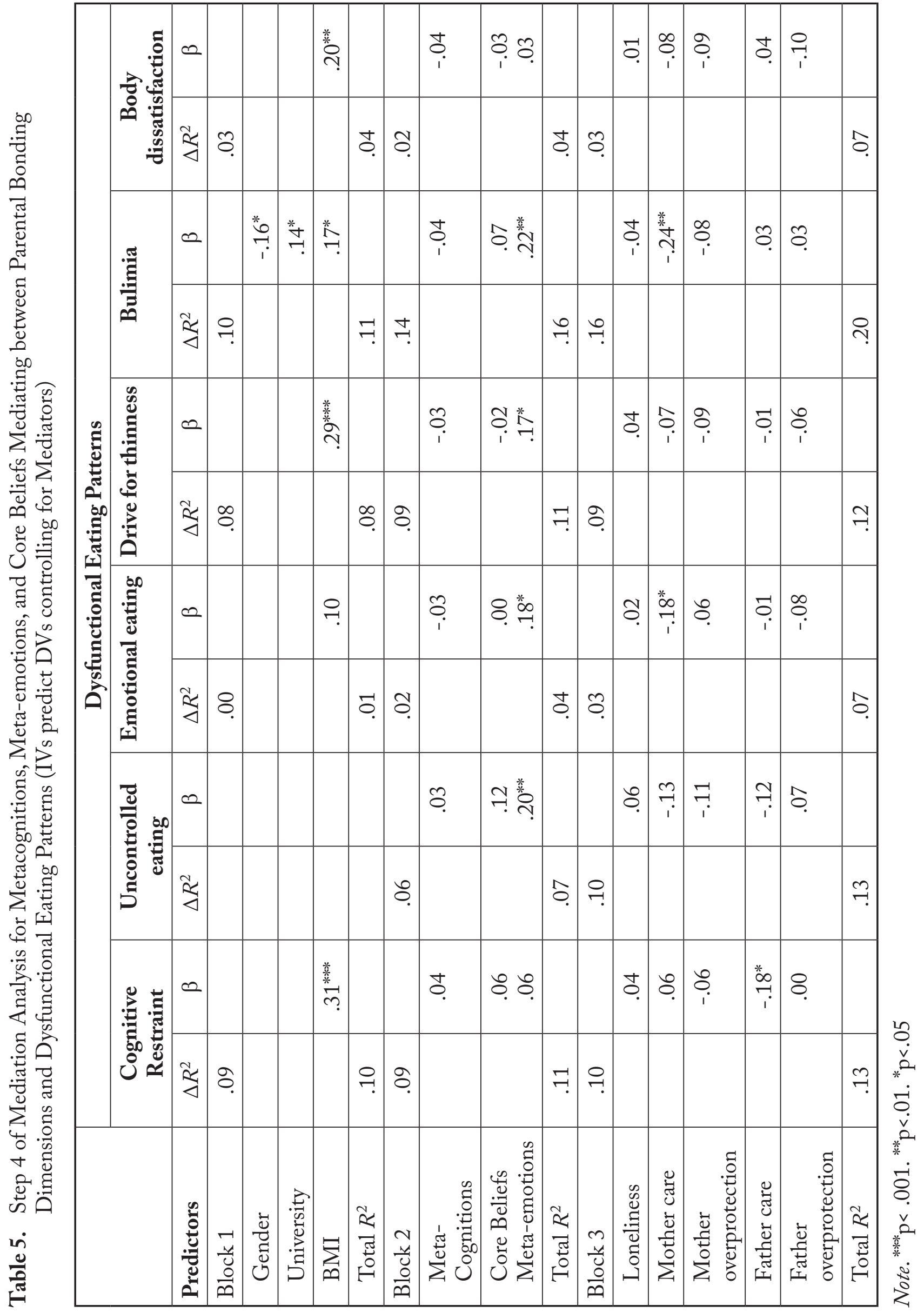


The findings of our study did not show any significant relationship between loneliness and dysfunctional eating patterns, and did not support the previous literature which suggested that early experiences with loneliness in childhood may set the stage for developing eating disorders. A 2002 study found women having a history of anorexia (binge/purge type) and bulimia reported of having more intense feelings of loneliness in their childhood as compared to controls (Troop \& Bifulco, 2002). Studies also show that loneliness in college students has a negative relationship with self-rated physical attractiveness and self-esteem (Stephan, Fath, \& Lamm, 1988) which ultimately resulted in self-rated physical attractiveness and negative perceptions toward oneself, especially with regard to how an individual evaluates his/her body, sexuality, appearance, and overall health (Goswick \& Jones, 1981), but none such findings emerged in the present study. One of the reasons for this result could be that in many Pakistani cultures, the prevalent family structure seldom lets an individual experience loneliness. The cultures encourage social meetings and gatherings and family system binds individuals with one another, increasing physical proximity as well as codependency. In this way, individuals may not experience loneliness as readily. Moreover, the concept of having meals together with the family may prevent one from establishing any negative association with food. This discipline ensures smooth operations of many household activities. On the contrary, Western cultures are known to encourage autonomy and liberation. A byproduct of this freedom may be confusion and lack of prior knowledge which may result in faulty lifestyle patterns.

The population evaluated in the present study was undergraduate university students. Incidence of dysfunctional eating was found in the sample. The prevalence and incidence of disordered eating patterns in Pakistan are more frequent compared to rates of fully diagnosed eating disorders. Previous research conducted in Pakistan also support this finding. The results of a study conducted by Muazzam and Khalid (2008) found that some sort of disordered eating behavior is manifested by 45 to $50 \%$ of Pakistani college students. Another study by Pelletier and Dion (2007) confirmed previous findings that the more females perceive sociocultural pressures about body image, the more they internalize society's beliefs about obesity and thinness, which was further linked to elevated levels of dissatisfaction with body image.

Findings of the present study do not support previous literature on metacognitions acting a mediator. As for its relationship with dysfunctional eating, only the cognitive confidence domain of metacognitions showed significant relationships with almost all patterns of dysfunctional eating. Kausar and Chohan (2015) hypothesized that there is likely to be an association between metacognitions, meta-emotions, and dysfunctional eating. Their study sample comprised 200 university students from two public sector universities of Lahore, Pakistan. The findings of their study revealed that emotional eating had a significant positive association with cognitive confidence and cognitive self-consciousness (dimensions of metacognitions) among men and with cognitive confidence and invalid emotions (dimension of meta-emotions) among women. Metacognitive components have also been observed to aid in the initiation and sustainment of psychopathology; these are manifested in ineffective response styles such as avoidance or suppression (Wells, 2000). According to previous research in which studies aimed to determine if metacognitions differ across typical and atypical anorexia in a non-clinical sample, and whether metacognitions simultaneously predict drive for thinness; it was found that despite having a healthier weight, the atypical group was as severely affected by drive for thinness and dysfunctional metacognitions as the typical group because metacognition simultaneously predicted drive for thinness in both groups (Davenport, Rushford, Soon, \& McDermott, 2015). The present research also failed to support the aforementioned finding and could not find a significant relationship between metacognition and drive toward thinness in general. 
Findings of the current study showed high standards for oneself and self-loathing to be the core beliefs prevalent in almost all patterns of dysfunctional eating, except their role as a mediator between parental bonding and patterns of dysfunctional eating was not supported. In the literature chronicling the prevalence of core beliefs in eating disordered individuals, Dingemans, Spinhoven and van Furth (2006) concluded in a study that individuals with eating disorders possess certain core beliefs but their beliefs were not found to be directly related to eating, shape, or weight. Imperatively, more severe maladaptive core beliefs were found to be associated with a greater frequency of purging and fasting behaviors. Hence, it was proposed that purging and fasting must be established as significant clinical markers.

Supporting past research, the present study found 'invalid and meaningless', 'useless', and 'shameful and irrational beliefs' about emotions to be significantly related with dysfunctional eating patterns. The mediation effect of meta-emotions between parental bonding and dysfunctional eating patterns could not be established in our study. According to the literature, beliefs about inability to accept negative emotions are a kind of excessively high standards or perfectionism regarding one's emotional expression and experience. In pathologies like anorexia, along with such attitudes to emotions, it is observed that more general perfectionistic beliefs may also occur wherein the individual believes that inability to meet high standards is equivalent to being a failure. This type of conditionally accepting oneself is considered to be a type of negative perfectionism (Schmidt \& Treasure, 2006).

\section{Limitations}

Limitations for the current research includes study participants reporting the questionnaire protocol to be too lengthy. It may be assumed that fatigue might have interfered with full responses. Another limitation may be strong cultural barriers between the Pakistani sample and western conventions, and possibly traditional clothing of South Asian populations. Varying beauty conventions and dress styles may be a factor in the demand for thinness across cultures.

As in many other cultures, South Asian cultures use food as a major facilitator of hosting and comfort. The concept of "portion control" is not a popular convention and many people eat usually to their fill. The generalizability of the research is limited because it drew from a collegiate pool in one Pakistani city. Other cities could not be accessed due to limited resource and time constraints.

\section{Suggestions for Future Research}

A revised, precise, and succinct questionnaire protocol should be designed to consume less time and energy on part of the study participant. It is also recommended that the data should be collected from other cities as well so that it can represent diverse culture and eating patterns across Pakistan.

\section{Implications of the Study}

The findings of this research can help in understanding possible associations with dysfunctional eating in the university students of one of Pakistan's largest city, Lahore, and might offer useful insights in cultural differences that may be of interest for those designing intervention strategies. It can further assist the practitioners in understanding eating attitudes of university students unique to a specific geographical area. 
The results of the study can be added to the existing literature on dysfunctional patterns of eating among Pakistani university students and can further open avenues to explore other relevant diversions of the same models. Furthermore, the study findings can be helpful in encouraging social and media norms from promoting very thin, unhealthy ideal body types. Also, the study of parental bonding and the role of parents in general as a predictor of dysfunctional eating tendency in their children will likely be revisited as a result.

\section{Conclusion}

The study aimed to find a relationship between loneliness, parental bonding (mother care, mother overprotection, father care, and father overprotection) and patterns of dysfunctional eating (uncontrolled eating, cognitive restraint, emotional eating, drive towards thinness, bulimia, and body dissatisfaction). The role of metacognitions, meta-emotions, and core beliefs as mediators between parental bonding and patterns of dysfunctional eating was also hypothesized. Results showed that parental bonding diversions had significant relationships with patterns of dysfunctional eating. No relationship was found between loneliness and patterns of dysfunctional eating, nor were metacognitions, meta-emotions, and core beliefs established as mediators between parental bonding and patterns of dysfunctional eating. However, a significant positive relationship between meta-emotions and almost all patterns dysfunctional eating (except body dissatisfaction and cognitive restraint) did emerge.

\section{References}

Aldao, A., Nolen-Hoeksema, S., \& Schweizer, S. (2010). Emotion-regulation strategies across psychopathology: A meta-analytic review. Clinical Psychology Review, 3o(2), 217-237. doi:10.1016/j.cpr.2009.11.004

APA (2000). Diagnostic and Statistical Manual of Mental Disorders, Text Revision (4th ed.). Washington, D.C: Author.

Anwar, T. \& Fatima, I. (2012). Risk Factors of Academics Stress and Health Risk Behaviors in Intermediate Students (Unpublished M.Phil thesis). University of the Punjab.

Arcelus, J., Mitchell, A. J., Wales, J., \& Nielsen, S. (2011). Mortality rates in patients with anorexia nervosa and other eating disorders: a meta-analysis of 36 studies. Archives of general psychiatry, $68(7), 724-731$.

Buchanan, N., Bluestein, B., Nappa, A., Woods, K., \& Depatie, M. (2013). Exploring gender differences in body image, eating pathology, and sexual harassment. Body Image, ro(3), 352-360. http:// dx.doi.org/10.1016/j.bodyim.2013.03.004

Calam, R., Waller, G., Slade, P., \& Newton, T. (1990). Eating disorders and perceived relationships with parents. International Journal of Eating Disorders, 9, 479-485.

Canetti, L., Bachar, E., Galili-Weisstub, E., De-Nour, A. K., \& Shalev, A. Y. (1997). Parental bonding and mental health in adolescence. Adolescence, $32(126), 381$.

Chohan, F. \& Kausar, R. (2015). Gender Differences in Dysfunctional Eating and Metacognitive Beliefs (M.Phil). University of the Punjab.

Choudry, I. \& Mumford, D. (1992). A pilot study of eating disorders in Mirpur (Pakistan) using an Urdu version of the eating attitudes test. Int. J. Eat. Disord., II(3), 243-251.http://dx.doi. org/10.1002/1098-108x(199204)11:3 < 243::aid-eat2260110307 > 3.0.co;2- p 
Clausen, L., Rosenvinge, J., Friborg, O., \& Rokkedal, K. (2010). Validating the Eating Disorder Inventory-3 (EDI-3): A Comparison Between 561 Female Eating Disorders Patients and 878 Females from the General Population. Journal Of Psychopathology And Behavioral Assessment, 33(1), 101-110. http://dx.doi.org/10.1007/s10862-010 9207-4.

Cooper, M., Grocutt, E., Deepak, K., \& Bailey, E. (2007). Metacognition in anorexia nervosa, dieting and non-dieting controls: A preliminary investigation. British Journal Of Clinical Psychology, 46(1), 113-117. http://dx.doi.org/10.1348/014466506x115245

Cooper, M., \& Burrows, A. (2001). Underlying assumptions and core beliefs related to eating disorders in the mothers of overweight girls. Behavioural And Cognitive Psychotherapy, 29(02). doi:10.1017/ s1352465801002028

Coopers, M. J., Rose, K. S., \& Turner, H. (2006). Specific content of core beliefs and schema in adolescent girls high and low in eating disorder symptoms. Eating behaviors, 7(1), 27-35.

Costarelli, V. \& Patsai, A. (2012). Academic examination stress increases disordered eating symptomatology in female university students. Eat Weight Disord 17(3):164-169.

Davenport, E., Rushford, N., Soon, S., \& McDermott, C. (2015). Dysfunctional metacognition

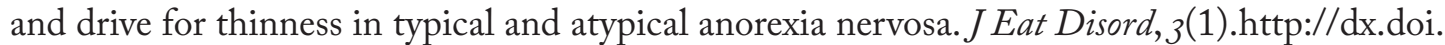
org/10.1186/s40337-015-0060-4

Dingemans, A., Spinhoven, P., \& Van Furth, E. (2006). Maladaptive core beliefs and eating disorder symptoms. Eating Behaviors, 7(3), 258-265. doi:10.1016/j.eatbeh.2005.09.007

Fairchild, H. \& Cooper, M. (2010). A multidimensional measure of core beliefs relevant to eating disorders: Preliminary development and validation. Eating Behaviors, $I I(4), 239-246$. http://dx.doi. org/10.1016/j.eatbeh.2010.05.004

Fassino, S., Amianto, F., Rocca, G., \& Dagga, G. (2010). Parental bonding and eating psychopathology in bulimia nervosa: personality traits as possible mediators. Epidemiol Psichiatr Soc., 19(3), 214-22.

Filaire, E., P. Treuvelot, et al. 2012. Relationship between eating-behavior disorders and psychological parameters in male first-year physical education students. Int J Sport Nutr Exerc Metab 22(5): 383-391.

Fontaine, K., Redden, D. T., Wang, C., Westfall, A. O., \& Allison, D. B. (2003). Years of Life Lost Due to Obesity. JAMA, 289(2), 187. doi:10.1001/jama.289.2.187-193.

Frost, J. (2013). What Are the Effects of Multicollinearity and When Can I Ignore Them?. Blog.minitab. com. Retrieved 5 December 2016, fromhttp://blog.minitab.com/blog/adventures-in-statistics/ what-are-the-effects-of- multicollinearity-and-when-can-i-ignore-them

Garner, D. M., Olmsted, M. P., \& Polivy, J. (1983). Development and validation of a multi dimensional eating disorder inventory for anorexia nervosa and bulimia. International Journal of Eating Disorders, $2,15-34$.

Goswick, R. A., \& Jones, W. H. (1981). Loneliness, self-concept, and adjustment. Journal of Psychology, Iо7, 237-241.

Groesz, L. M., Levine, M. P., \& Murnen, S. K. (2002). The effect of experimental presentation of thin images on body satisfaction: A meta-analytic review. International Journal of Eating Disorders, 3I, $1-16$.

Heesacker, R., \& Neimeyer, G. (1990). Assessing object relations and social cognitive correlates of eating disorders. Journal of Counselling Psychology, 37, 521-526.

Hussain, M. \& Kausar, R. (2016). Psychological Constructs and Dysfunctional Eating in University Students (Unpublished MS thesis). University of the Punjab. 
Jones, C., Harris, G., \& Leung, N. (2005). Core beliefs and eating disorder recovery. European Eating

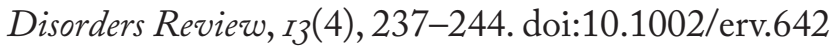

Karlsson, J., Persson, L., Sjöström, L., \& Sullivan, M. (2000). Psychometric properties and factor structure of the Three-Factor Eating Questionnaire (TFEQ) in obese men and women. Results from the Swedish Obese Subjects (SOS) study. Int J Obes Relat Metab Disord, 24(12), 1715-1725. http://dx.doi.org/10.1038/sj.ijo.0801442

Katsounari, I. \& Zeeni, N. (2012). Preoccupation with Weight and Eating Patterns of Lebanese and Cypriot Female Students. Psychology, o3(06), 507-512. http://dx.doi.org/10.4236/ psych.2012.36073

Kendrick, M. (2015). Why being 'overweight' means you live longer: The way scientists twist the fact. Retrieved October 18, 2015 from http://www.independent.co.uk.

Konstantellou, A., \& Reynolds, M. (2010). Intolerance of uncertainty and metacognitions in a non-clinical sample with problematic and normal eating attitudes. Eating Behaviors, $I I(3)$, 193-196. doi: 10.1016/j.eatbeh.2010.01.003

Leung, N., Waller, G., \& Thomas, G. (2000). Outcome of group cognitive-behaviour therapy for bulimia nervosa: The roles of core beliefs. Behaviour Research and Therapy, 38, 145-156.

Lucas, A. R., Crowson, C. S., O’ Fallon, W. M., \& Melton, L. J. (1999). The ups and downs of anorexia nervosa. International Journal of Eating Disorders, 26(4), 397-415.

Masheb, R. M., \& Grilo, C. M. (2006). Emotional overeating and its associations with eating disorder psychopathology among overweight patients with binge eating disorder. International Journal of Eating Disorders, 39, 141-146.

McDermott, C. J., \& Rushford, N. (2011). Dysfunctional metacognitions in anorexia nervosa. Eating and Weight Disorders, I6(1), e49-e55.

Memon, A. A., S. E. Adil, et al. 2012. Eating disorders in medical students of Karachi, Pakistan-a cross-sectional study. BMC Res Notes 5: 84.

Muazzam, A. \& Khalid, R. (2011). Development and Validation of Disordered Eating Behavior Scale: Identification, Prevalence, and Difference with Clinically Diagnosed Eating Disorders. Pakistan Journal Of Psychological Research, 26(2), 127-148.

Muazzam, A., \& Khalid, R. (2008). Disordered eating behaviors: An overview of Asian cultures. Journal of Pakistan Psychiatric Society, 5(2), 76-81.

Mumford, D., Whitehouse, A., \& Choudry, I. (1992). Survey of eating disorders in English -

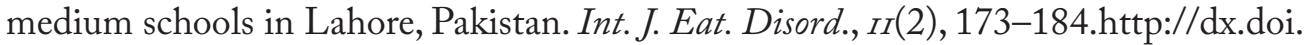
org/10.1002/1098-108x(199203)11:2 < 173::aid-eat2260110208 > 3.0.co;21

Parker, G., Tupling, H., \& Brown, L. (1979). A parental bonding instrument. British Journal of Medical Psychology, 52, 1-10.

Parker, G. (1983). Parental overprotection. New York: Grune \& Stratton.

Parker, G. (1990). The parental bonding instrument. Social Psychiatry And Psychiatric Epidemiology, 25(6), 281-282. http://dx.doi.org/10.1007/bf00782881

Pelletier, L. \& Dion, S. (2007). An Examination of General and Specific Motivational Mechanisms for the Relations Between Body Dissatisfaction and Eating Behaviors. Journal Of Social And Clinical Psychology, 26(3), 303-333.http://dx.doi.org/10.1521/jscp.2007.26.3.303

Russell, D. (1996). UCLA Loneliness Scale (Version 3): Reliability, validity, and factor structure. Journal of Personality Assessment, 66, 20-40. 
Rimes, K., \& Chalder, T. (2010). The Beliefs about Emotions Scale: Validity, reliability and sensitivity to change. Journal Of Psychosomatic Research, 68(3), 285-292. http://dx.doi.org/10.1016/j. jpsychores.2009.09.014

Safdari S, Khoramdel K, \& Kamranian E. (2013). The role of metacognitive beliefs in eating disorders. Journal of Social Issues E Humanities. I:96-9.

Schmidt, U. \& Treasure, J. (2006). Anorexia nervosa: Valued and visible. A cognitive interpersonal maintenance model and its implications for research and practice. British Journal Of Clinical Psychology, 45(3), 343-366.http://dx.doi.org/10.1348/014466505x53902

Shaikh, M. A. \& Kayani, A. (2014). Detection of eating disorders in 16-20 year old female students—perspective from Islamabad, Pakistan. J Pak Med Assoc 64(3): 334-336.

Sorenson, P., \& Strodl, E. (2015). Gender differences in metacognitions, meta-emotions, and dysfunctional eating. Submitted for review.

Stephan, E., Fäth, M., \& Lamm, H. (1988). Loneliness as related to various personality and environmental measures: Research with the German adaptation of the UCLA loneliness scale. Social Behavior \& Personality: An International Journal, $16,169-174$.

Stewart, W. (2004). The role of perceived loneliness and isolation in the relapse from recovery in patients with anorexia and bulimia nervosa. Clinical Social Work Journal, 32, 185-196.

Stice, E. (2001). A prospective test of the dual-pathway model of bulimic pathology: Mediating effects of dieting and negative affect. Journal of Abnormal Psychology, IIO, 124-135.

Stice, E. (1994). A review of the evidence for a sociocultural model of bulimia nervosa and an exploration of the mechanisms of action. Clinical Psychology Revierw, I4, 633-661.

Stuart, G. W., Laraia, M. T., Ballenger, J. C., \& Lydiard, R. B. (1990). Early family experiences of women with bulima and depression. Archives of Psychiatric Nursing, 4, 43-52.

Suhail, K., \& Nisa, Z. (2002). Prevalence of eating disorders. Journal of Eating and Weight Disorders, $7(2), 131-138$.

Tata, P., Fox, J., \& Cooper, J. (2001). An investigation into the influence of gender and parenting styles on excessive exercise and disordered eating. European Eating Disorders Review, 9(3), 194-206.

Tchanturia, K., Lloyd, S., \& Lang, K. (2013). Cognitive remediation therapy for anorexia nervosa: Current evidence and future research directions. Int. J. Eat. Disord., 46(5), 492-495. http://dx.doi. org/10.1002/eat.22106

Troop, N. A., \& Bifulco, A. (2002). Childhood social arena and cognitive sets in eating disorders. British Journal of Clinical Psychology, 41, 205-213.

Turner, H., Rose, K., \& Cooper, M. (2005a). Schema and parental bonding in overweight and nonoverweight female adolescents. Int J Obes Relat Metab Disord, 29(4), 381-387. doi:10.1038/ sj.ijo.0802915

Turner, H., Rose, K., \& Cooper, M. (2005b). Parental bonding and eating disorder symptoms in adolescents: The meditating role of core beliefs. Eating Behaviors, 6(2), 113-118. doi:10.1016/j. eatbeh.2004.08.010

Tuschen-Caffier, B., \& Vögele, C. (1999). Psychological and physiological reactivity to stress: An experimental study on bulimic patients, restrained eaters and controls. Psychotherapy and Psychosomatics, 68, 333-340.

Vann, A., Strodl, E., \& Anderson, E. (2013). Thinking about internal states, a qualitative investigation into metacognitions in women with eating disorders. J Eat Disord, I(1), 22. doi:10.1186/20502974-1-22. 
Walters, E. E., \& Kendler, K. S. (1995). Anorexia nervosa and anorexic-like syndromes in a population-based female twin sample. American Journal of Psychiatry, 152, 64-71.

Wells, A. (2009). Introduction to the special section on metacognition. International Journal of Cognitive Therapy, 2(2), 105-106. doi: 10.1521/ijct.2009.2.2.105

Wells, A. (2000). Emotional Disorders \& Metacognition: Innovative Cognitive Therapy. Chichester: John Wiley \& Sons Ltd. 